\title{
Optical Networks Control and Management
}

\author{
Jing Wu • Lena Wosinska • Gangxiang Shen • \\ Salvatore Spadaro
}

Received: 26 February 2014/Revised: 22 March 2014/ Accepted: 26 March 2014/

Published online: 2 April 2014

(C) Her Majesty the Queen in Rights of Canada 2014

Control and management play a key role in enabling optical networks to efficiently and smoothly carry user data. In the recent years, there has been much advance in the areas, ranging from the traditional Generalized Multi-Protocol Label Switching (GMPLS) to today's Path Computation Element (PCE)-based technology. In addition, elastic, spectrum-efficient and gridless optical networks, reduction of energy consumption, and Software Defined Networking (SDN) in optical networks are gaining attention from both research community and network operators. Consequently, network control and management system that can efficiently allocate optical fiber spectrum resources and support energy-efficient operation will be another exciting near-term research focus. The future optical network management and control plane is expected to be more versatile, scalable, automatic, and resource-efficient.

\footnotetext{
J. Wu (

Communications Research Centre (CRC), 220 Denali Way, Stittsville, ON K2S 0N1, Canada e-mail: jingwu@ieee.org
}

L. Wosinska

Optical Networks Lab, Department of Communication Systems, School of ICT, KTH Royal Institute of Technology, Isafjordsgatan 22 Electrum, 229, 16440 Kista, Sweden e-mail: wosinska@kth.se

G. Shen

Optical Network Technology Research Center (ONTRC), School of Electronic and Information Engineering, Soochow University, Shizi RD \#, Suzhou, Jiangsu Province, People's Republic of China

e-mail: shengx@suda.edu.cn

\section{S. Spadaro}

Optical Communications Group, Universitat Politècnica de Catalunya BarcelonaTech, Jordi Girona 1-3, D4-S108, 08034 Barcelona, Spain

e-mail: spadaro@tsc.upc.edu 
This special issue aims at providing an opportunity for researchers and engineers to share their latest research results and advances in optical network control and management. Three research papers are accepted after a review and revision process.

The integration of Passive Optical Networks (PONs) with next-generation wireless access networks is a promising solution for the access networks, where fiber infrastructure provides very high transmission capacity while wireless segment offers mobility for the users. Moreover, such an integration is a cost-effective way of backhauling the future wireless access networks. In paper "An Efficient Resource Allocation Mechanism for LTE-GEPON Converged Networks", C. Ranaweera, E. Wong, C. Lim, A. Nirmalathas, and C. Jayasundara proposed a resource allocation mechanism for LTE-GEPON (long term evolution-Gigabit Ethernet PON) converged networks that improves the Quality of Service (QoS) performance of the converged network.

More recently, multi-domain and multi-layer optical transport networks became a new research focus for optical networks control and management. In paper "Techniques and Benefits of Energy-Aware Load-Distribution in Multi-domain Translucent Wavelength Switched Optical Networks", A. Pagès, A. Buttaboni, G. Maier, D. Siracusa, J. Perelló, and S. Spadaro proposed a novel energy-aware interdomain connection provisioning mechanism for translucent Wavelength Switched Optical Networks, with the objective of performing end-to-end route computations while minimizing energy consumption.

PCE architecture has been defined for different scenarios such as multi-layer and multi-domain. PCE computes the routes based on the information in the Traffic Engineering Database (TED). TED is updated with the control plane information. In paper "Implementation and Assessment of Pre-Reservation mechanism for stateless PCE environments”, M. Cuaresma, V. López, O. González de Dios, F. Muñoz del Nuevo, and J. P. Fernández-Palacios demonstrated the importance of the prereservation mechanism in two scenarios: multiple PCEs architecture and massive restoration.

We would like to thank all the authors for their contributions to this special issue. We highly appreciate the valuable support of the individual reviewers through their great efforts in providing authors with constructive and timely feedback. To avoid conflict of interest, the paper by Pagès et al. (that includes one of the guest editors as a co-author) was assigned to an associate editor of the journal for handling by the editor-in-chief. We thank the anonymous associate editor for handling this paper for the special issue. Finally, we express our gratitude to Deep Medhi, Editor-in-Chief of this journal, for supporting the organization of this special issue.

Jing Wu is an Engineer in Environment Canada, Science and Technology Branch. He was a Research Scientist at the Communications Research Centre Canada between 2001 and 2013. Currently, he is also appointed as an Adjunct Professor at the University of Ottawa, School of Electrical Engineering and Computer Science. 
Lena Wosinska joined KTH Royal Institute of Technology in 1986, where she is currently a Professor in Telecommunication, heading the Optical Networks Lab (ONLab). She has been working in several EU projects and coordinating a number of national research projects. Her research interests include fiber access networks, energy efficient optical networks, photonics in switching, optical network management, reliability and survivability.

Gangxiang Shen received his Ph.D. degree from University of Alberta, Canada. Currently he is a professor with Soochow University, China. His research interests include spectrum and energy efficient optical networks. He was a guest editor of two IEEE JSAC special issues and is also an associated editor of IEEE/OSA JOCN.

Salvatore Spadaro received the M.Sc. and the Ph.D. Degrees in Telecommunications Engineering from Universitat Politècnica de Catalunya (UPC). His interests are in the fields of all-optical network solutions with emphasis on network control and management, network virtualization, intra/inter data centres interconnects, flex-grid technologies, energy efficiency, Software Defined Networking and virtual optical networks embedding. 